\title{
CUANTIFICACIÓN DE LOS GRADIENTES TÉRMICOS A NIVEL SUPERFICIAL A LO LARGO DEL RHEINLAND-PFALZ (RENANIA-PALATINADO, ALEMANIA)
}

\author{
Jesús RodRIGo COMINO
}

\begin{abstract}
RESUMEN
En este estudio se propone una secuencia metodológica apoyada en el cálculo de gradientes térmicos atmosféricos a nivel de superficie. El trabajo es aplicado dentro del estado del Rheinland-Pfalz (Renania-Palatinado, Alemania), donde se observan interesantes resultados que muestran la aparición de diferencias entre el estado termométrico de la atmósfera en las áreas más elevadas y los fondos de valle. Se aprecian inversiones térmicas en algunos intervalos de altitud y meses concretos, desajustes por culpa de factores externos y claves para favorecer una correcta ordenación del territorio orientada a unos usos determinados del suelo. Para definir los gradientes térmicos, se parte de un análisis clúster y el cálculo de estimaciones lineales representadas finalmente en gráficas con líneas de tendencia.
\end{abstract}

\begin{abstract}
A methodological sequence with calculations of atmospheric thermal gradients at surface level is proposed in this study. This methodology has been applied in the RheinlandPfalz state (Rhine-Palatinate, Germany), where we can observe interesting effects, such as the differences between the atmospheric state of high and valley areas. Thermic inversions at certain altitude intervals during particular months, thermic imbalances by external factors and keys for improving land management in different land uses have been detected. To define the thermal gradients, this paper will begin with a cluster analysis and calculations of linear estimates. Finally they will be represented in graphics with trend lines.
\end{abstract}

PALABRAS CLAVE: gradiente térmico vertical, Rheinland-Pfalz, temperaturas, estimación lineal y análisis cluster

KEYWORDS: vertical thermal gradients, Rheinland-Pfalz, temperatures, linear estimates and trend lines

\section{INTRODUCCIÓN}

$\mathrm{Al}$ analizar las condiciones climáticas de un territorio, el contraste entre paisajes pone de manifiesto la evidencia de que existen numerosos factores 
que revelan diferencias marcadamente variables (Senciales, 2007). Todos los factores que intervienen en que un clima se caracterice por una serie de contrastes u homogeneidades están totalmente interconectados y son dependientes entre sí. De esta forma, el estado de la atmósfera en un lugar y momento determinado será el resultado de la acción conjunta de factores como la latitud, las horas de sol, la nubosidad, el viento, la fisiografía, las masas de vegetación, los usos del suelo, el tipo de capa edáfica o la proximidad a centros de acción de estabilidad o inestabilidad.

La tierra es atravesada por radiaciones electromagnéticas de abajo-arriba y de arriba-abajo, actuando de forma determinante sobre la transformación en energía calorífica de las moléculas de la atmósfera, medida a través de la temperatura. Numerosos autores se han preocupado de definir diferentes capas concéntricas a lo largo del planeta (Strahler y Strahler, 1989; Gebhardt et alii, 2012): troposfera, estratosfera, ionosfera,... sin embargo, como apunta Demangeot (1989) "a estas alturas el cielo se hace negro y se encuentra muy lejos de la atmósfera del geógrafo", aunque no por ello no le interese su influencia sobre la relación entre el ser humano y el medio que habita, como sería el caso de los riesgos climáticos o el calentamiento global.

Esta línea, que toma como referencia para la gestión del territorio las condiciones térmicas de la atmósfera, puede estudiarse desde el análisis de las curvas de estado y gradientes térmicos verticales. Estas líneas marcan el crecimiento o decrecimiento termométrico de los diferentes intervalos altitudinales del aire próximas al suelo. Dichas variaciones de las temperaturas según diversos factores, de sobra conocidos (exposición, altitud, cercanía o lejanía de masas de agua, presencia o ausencia de vegetación o núcleos urbanos,...), ya han sido publicados en manuales clásicos sobre climatología como los de Escourrou (1957) o Flohn (1968). Estos autores ya confirman una variación aproximada de entre 3 y $5^{\circ} \mathrm{C}$ por cada $1.000 \mathrm{~m}$ que se ascienda o descienda (según aparezcan o no inversiones térmicas), y por tanto, remarcando las diferencias entre montaña y valle o una atmósfera condicionada o libre (Font Tullot, 1983). Sin embargo, estas circunstancias tampoco, obviamente, son ni homogéneas, ni constantes desde el suelo hasta los límites de la atmósfera con el espacio exterior (Gebhardt et alii, 2012). De ahí, que resulte interesante calcular esos descensos o aumentos en la temperatura en los espacios tanto ocupados por el hombre, como naturales, con objeto de comprender las causas de la distribución de estos sucesos.

Por último, es importante mencionar que la aparición de un gradiente vertical en la capa de la atmósfera que permanece en contacto con la superficie terrestre (troposfera) también está condicionada por la presión del vapor de agua (Pedelaborde, 1982; Strahler y Strahler, 1989), hecho que descubre el interés por el cálculo de estas cifras para llegar a la aproximación de los posibles 
factores térmicos e higrométricos antes, durante y después de posibles precipitaciones que condicionan los suelos desnudos, con vegetación o cultivados.

\section{2. ÁREA DE ESTUDIO}

El presente estudio se enmarca dentro de uno de los estados federados de la República Federal de Alemania denominado Rheinland-Pfalz y traducido al español como Renania-Palatinado. Esta región abarca alrededor de 20.000 $\mathrm{km}^{2}$ y está condicionada por la influencia de uno de los cursos fluviales más importantes de Europa por volumen de agua movilizado (más de $2.000 \mathrm{~m}^{3 /}$ seg) y longitud (alrededor de unos $1.300 \mathrm{~km}$ ): El Rin (Rhein). Colinda al W con los países de Bélgica y Luxemburgo, al NW con el estado de NordrheinWestfalen, al E con Hessen, al SE con Baden-Württemberg y al S con el estado de Saarland y Francia.

\section{MAPA 1}

GEOLOCALIZACIÓN DEL ESTADO DE RHEINLAND-PFALZ (RENANIA-PALATINADO, ALEMANIA)
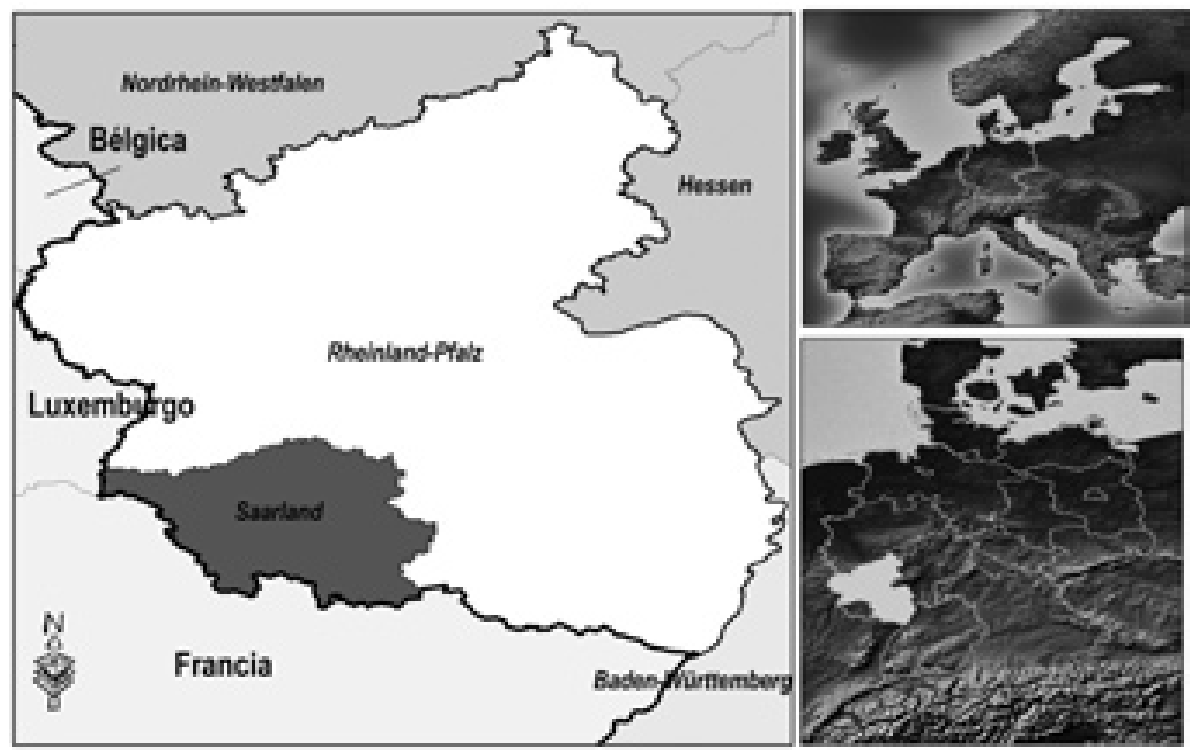

Los espacios naturales que se generan alrededor de los diferentes niveles de terrazas y valles fluviales de los afluentes del Rin albergan una gran heterogeneidad en sus paisajes. La topografía generadora de mayores contrastes termométricos (además de paisajísticos) se componen por materiales 
devónicos con pizarras altamente metamorfizadas (Rheinisches Schiefergebirge), litologías sedimentarias triásicas de areniscas masivas con tonos rojizos (Sandsteinen) y prominentes formaciones volcánicas emplazadas en la región de Eifel (www.lgb-rlp.de). Así, los contrastes más extremos entre montaña y valle podrían establecerse con los picos más elevados de esta región y los valles fluviales antes mencionados: Erbeskopf (816 m), An den Zwei Steinen o el Kahlheid (766 m ambos) y los ríos Rin (Rhein), Mosela (Mosel), Sarre (Saar) o Lahn.

Los núcleos poblacionales más destacados son las ciudades de Mainz (Maguncia) que es la capital de la región, Kaiserlautern, Trier (Treveris), Koblenz (Coblenza) y Ludwigshafen. Dichos espacios urbanos están conectados por paisajes altamente antropizados que parten desde las grandes obras ingenieriles de las autopistas, carreteras nacionales o de los canales y esclusas, que permiten la regulación del caudal de los grandes ríos y sus afluentes o el transporte de mercancías y personas. Además, son parte fundamental de la economía la comunión entre estas vías de comunicación y los usos agrarios e industriales relacionados con el sector vitivinícola (Gunzelmann, 2005; Petit et alii. 2012). Sobre este mercado y la distribución de la población, las diferencias regionales climáticas han supuesto un elevado condicionamiento que ha sido tenido en cuenta en la planificación del territorio (Unwin, 1996) al organizarse los grandes centros de transporte en los inminentes núcleos industriales y con población demandante suficiente para acoger la oferta creciente (Krämer, 2009). Las diferencias entre los períodos cálidos y fríos, diarios y mensuales, han provocado que consecuentemente dichas restricciones alteren la fluidez de una densidad de población que está totalmente mezclada con espacios ocupados de forma muy extensiva en las áreas periurbanas, que contrastan en la tipología edificatoria con las grandes metrópolis (Glaser, 2001).

\section{MÉTODOS Y MATERIALES}

En primer lugar, se han seleccionado un total de 56 estaciones climáticas (tabla 1) que poseen datos de temperaturas con un mínimo de 30 años completos (1961-1990 y 1981-2000), premisa principal que recomendó la $O M M$ en 1935 (CEOTMA, 1984). Dichos puntos se encuentran enmarcados en el estado de Rheinland-Pfalz (Renania-Palatinado, Alemania) y han sido obtenidos desde la web oficial dedicada al clima del Bundesministerium für Verkehr, Bau und Stadtentwiclung (Ministerio Federal de Transportes, Obras Públicas y Desarrollo Urbano) de libre acceso hasta la fecha de la realización de este trabajo (www.dwd.de). 


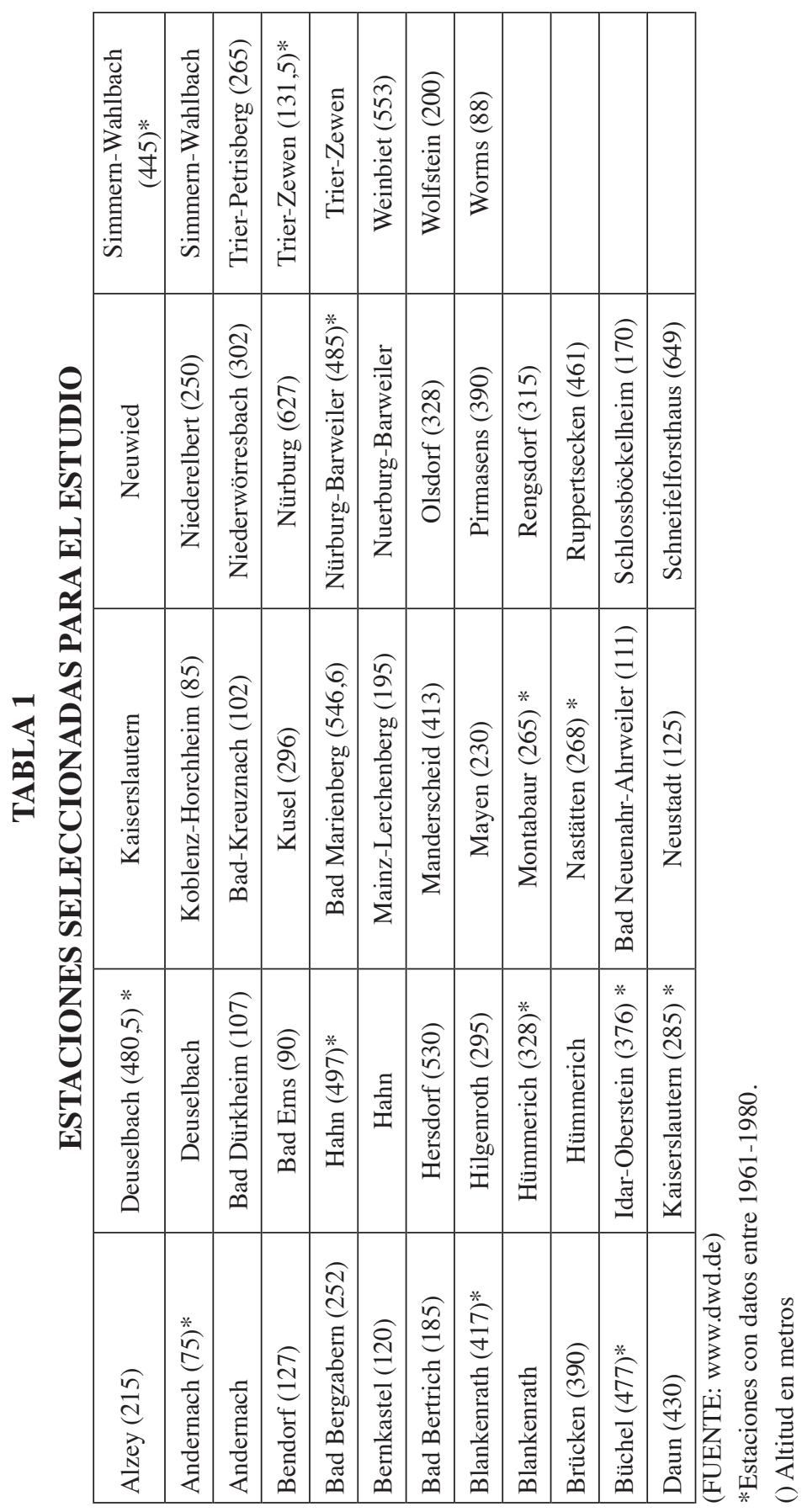

(C) Baetica. Estudios de Arte, Geografía e Historia, 35, 2013, 75-97. ISSN: 0212-5099

Facultad de Filosofía y Letras, Universidad de Málaga. Campus de Teatinos, E-29071 Málaga (España) 
El siguiente paso será ordenar las estaciones según intervalos de 100 m.s.n.m. y realizar para cada uno un análisis clúster (de tipo conglomerado jerárquico) con el software SPSS Statistics 19, apoyándose en el cálculo de la distancia euclidiana para encontrar grupos homogéneos según las variables utilizadas (altitud, temperaturas medias mensuales y anuales). Estas se sitúan en un plano imaginario donde se calcula la longitud del segmento de una recta que tiene por extremos las variables seleccionadas. De esta forma, se detectan con una mayor probabilidad las estaciones que establecerán desajustes. Para poder analizar dichos resultados, se representan en un dendrograma y una tabla de distancias, pudiéndose observar qué estaciones alterarían el cálculo posterior del gradiente por su disimilaridad o las que son más parecidas en su comportamiento termométrico mensual y anual globalmente (Figura 1):

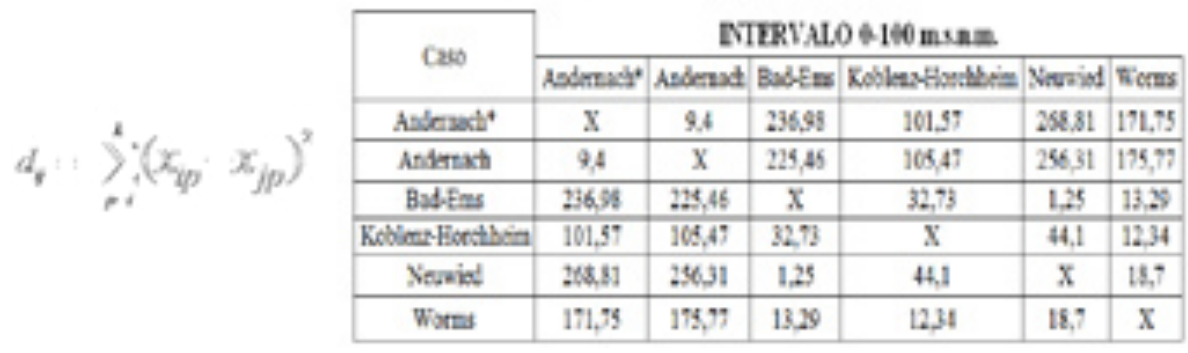

Figura 1. Fórmula de la distancia euclidiana y ejemplo de tabla de distancias con las estaciones seleccionadas

A partir de aquí, se calcula por intervalos el gradiente de la atmósfera a nivel de superficie con el dato de la temperatura media de cada mes y anual. Para este estudio, se ha optado por la realización de estimaciones lineales y cálculos de la intersección con el eje, para finalmente ser ratificadas las cifras con el cálculo del coeficiente de correlación. Este último paso, se considera vital dentro de este proceso porque deben ser detectados los condicionantes que provienen de los diferentes desajustes o factores externos condicionantes (inversiones térmicas, presencia de bosques o masas de agua, zonas urbanas,...). Para que un ajuste sea idóneo, debe situarse $R^{2}$ en cifras mayores a 0,9 con el uso de, al menos, tres estaciones para su obtención, ya que con dos puntos de medición solamente la correlación sería, con toda lógica, perfecta $\left(R^{2}=1\right)$ sin adecuarse a más realidades del territorio que hacen que el gradiente posea inflexiones. Así, esta primera parte mostrará la situación termométrica de la atmósfera en los territorios comprendidos entre las estaciones utilizadas en momentos determinados del mes.

Por último, para representar los resultados y extenderlos de forma generalizada para todo el estado del Rheinland-Pfalz, se añaden los valores a grá- 
ficas a las que se adjuntan líneas de tendencias lineales para examinar cuál es el comportamiento más ajustado para cada mes. De esta forma se determinan posibles comportamientos y desajustes generales en el territorio estudiado: descenso de la temperatura con la altitud, inversiones térmicas, inflexiones en los valores termométricos según altitud y meses concretos... Así, quedará diseñada la información estadística principal para un estudio más exhaustivo posterior, donde se pretenderá identificar en cada mes qué factor o desajuste implica sobre el territorio los cambios en las temperaturas, todo ello, analizado directamente sobre la cartografía y el uso de los SIG.

\section{RESULTADOS}

\subsection{Análisis clúster para la agrupación de estaciones climáticas}

Como paso previo al cálculo de los gradientes térmicos a nivel de superficie por intervalos de 100 metros, se ha realizado un análisis clúster (conglomerado jerárquico) con el software SPSS Statistics 19 apoyado en la fórmula matemática de la distancia euclidiana anteriormente explicada. Como resultado, al incluir como variables las doce temperaturas medias mensuales, la anual y la altitud se han organizado en diferentes subgrupos (clústeres) las distintas estaciones según su homogeneidad. A continuación, se presentan los dendrogramas (Figuras 2, 3, 4, 5, 6, y 7) con la relación entre estaciones por cada intervalo y un breve comentario que se ha apoyado también en la observación de la tabla de distancias obtenidas (ejemplo en la Figura 1):

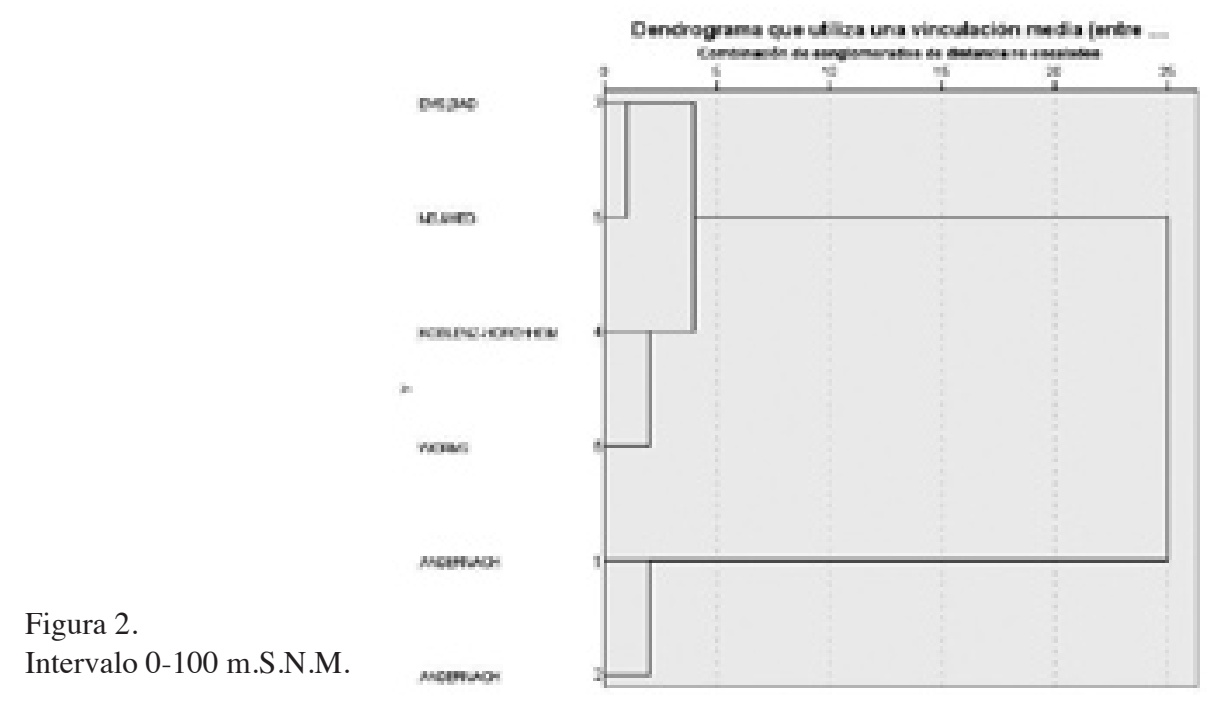


Para este primer intervalo, se puede observar un clúster diferenciado compuesto por las estaciones de Ems, Koblenz-Horcheim y Neuwied, que ocasionalmente también mantiene una buena correlación con Worms. Quedarían en otro clúster las dos estaciones de Andernach con unas condiciones semejantes entre sí, pero con desajustes respecto a las demás.

Figura 3.

Intervalo 100-200 m.S.N.M

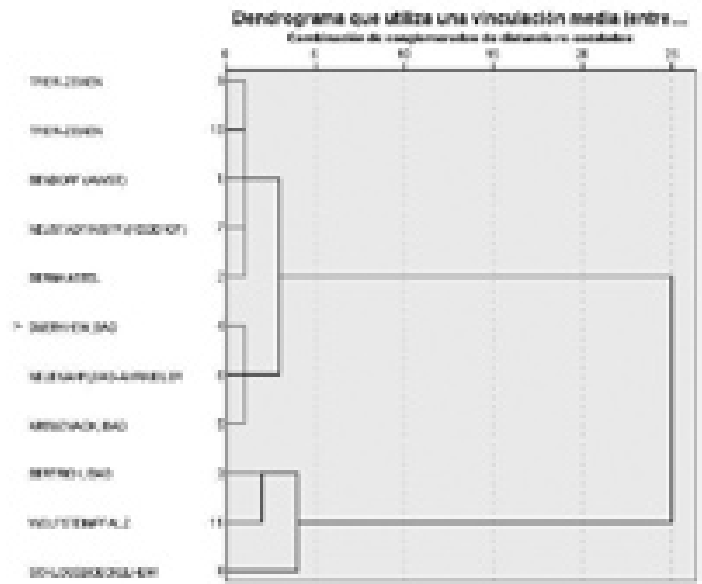

Para el intervalo 100-200 m, las estaciones que aparecen con una relación de distancia más fuerte serían las de Trier-Zewen, Neustadt, Bendorf y Bernkastel. En un siguiente clúster aparecerían Bad Dürkheim, Bad NeunahrAhrweiler y Bad Kreuznach. Y por último con menos relación con las demás y entre ellas las de Bad Bertrich, Wolfstein y Schlossböckelheim.

Figura 4.

Intervalo 200-300 m.S.N.M

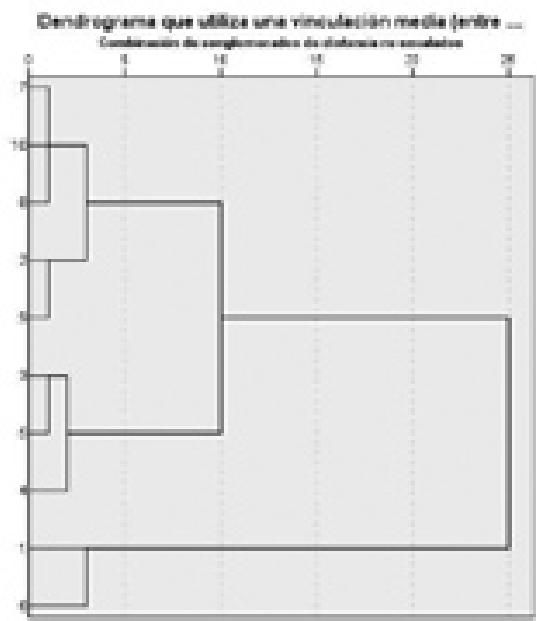


Para el intervalo comprendido entre 200-300 m, el clúster más fuerte lo conforman Trier-Petrisberg, con Montabur y Nastäten, con algo menos de relación con las de Berzgabern y Niederelbert. Las estaciones de Hilgenroth y Kusel mantienen una fuerte relación, y solo estas tienen algo de correlación con la de Kaiserlautern (que permanece con condiciones térmicas muy diferentes al resto de las demás del intervalo). Por último, las mediciones en Alzey y Mayen están unidas entre sí, pero muy separadas del resto de estaciones, lo que luego llevará a desajustes interesantes en el cálculo de los gradientes como se verá.

Figura 5.

Intervalo 300-400 m.S.N.M

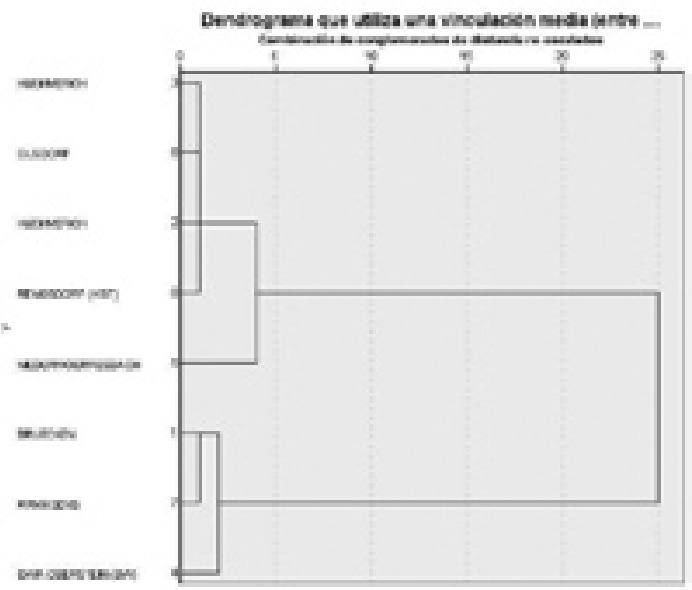

Figura 6.

Intervalo 400-500 m.S.N.M

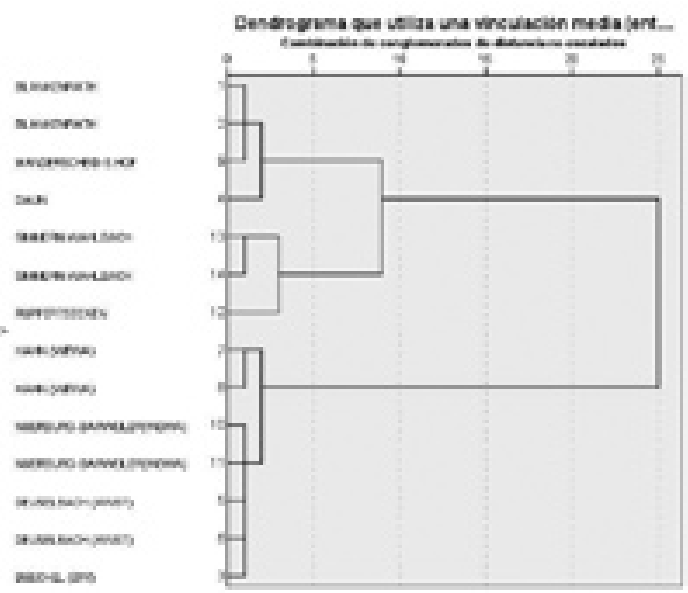


Las relaciones en el intervalo de la figura 5 muestran desajustes importantes, porque quedan dos estaciones separadas del resto y solo unidas a clústeres más grandes como son las de Niederwörresbach e Idar-Oberstein. Por otra parte, conformando grupos más homogéneos se obtienen las dos de Hümmerich, Oldorf y Rengsdorf; y Brücken y Pirmasens por otro. Estas diferencias podrán generar situaciones de inestabilidad en gradientes a nivel de superficie o facilitar inversiones térmicas.

Para el intervalo 400-500 m, con un número más elevado de estaciones, se observan pequeños clústeres que pueden alterar los cálculos posteriores y dar lugar a la obtención de situaciones diversas con desajustes termométricos. Quedarían aisladas con relaciones no tan claras las dos de Deuselbach, Büchel, Rupperstein y Daun. En un siguiente nivel, se forman clústeres de dos estaciones como los de Nürburg-Barweiler, los de Hahn y Simmern-Wahlbach. Por último, algo más cercanas en las distancias del dendrograma y la tabla se hallan las dos de Blankenrath y la de Manderscheid.

Figura 7. Intervalo >500 m.S.N.M

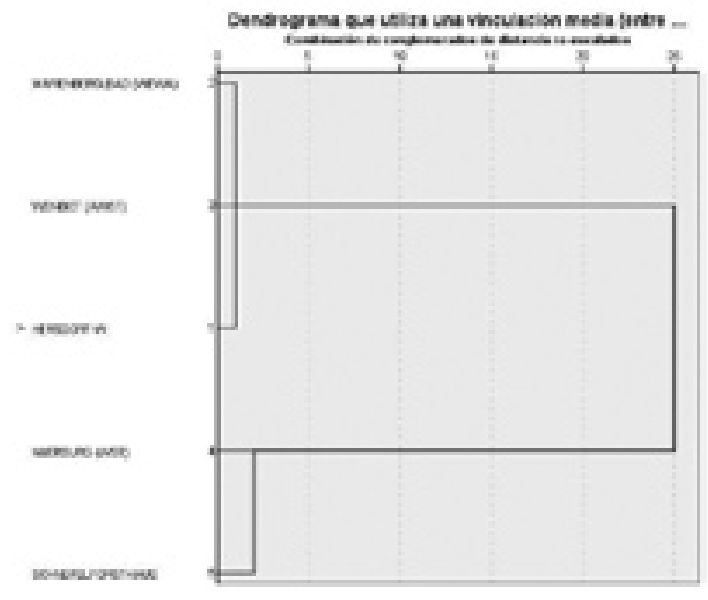

Para el último intervalo, se han agrupado las últimas estaciones restantes para tener suficientes datos para el cálculo de los gradientes, como ya se adelantó, pues es necesario contar con tres o más centros con información termométrica disponibles. Así, se puede observar claramente la formación de dos clústeres diferenciados, el primero entre Bad Marienberg, Weinbet y Hersdorf, y un segundo con Nürburg y Schneifelforsthaus.

\subsection{Cálculo del gradiente vertical general y curva de tendencia}

En primer lugar, se ha considerado incluir el cálculo del gradiente vertical general (sin inflexiones por intervalos que sí mostrarán más adelante las cur- 
vas de estado atmosférico) que teóricamente debería aplicarse en esta región del Rheinland-Pfalz para las extrapolaciones térmicas que se quisieran realizar si se dispusiera de un territorio con una "atmósfera libre" de influencias de factores externos como la definía Font Tullot (1983). Esto quiere decir que más que obviarse la influencia de las masas de agua, bosques, áreas urbanas o cultivadas, serían integrados en un cálculo muy general. Por ese motivo, para precisar el comportamiento por intervalo de altitud y territorio comprendido entre estaciones climáticas, sería necesario analizar mes por mes cada comportamiento de la curva de estado atmosférica, en vez de un gradiente vertical con una atmósfera libre. A continuación, se presenta la figura 8 con el cálculo realizado con las estaciones que dan un $\mathrm{R}^{2}$ más cercano a 1:

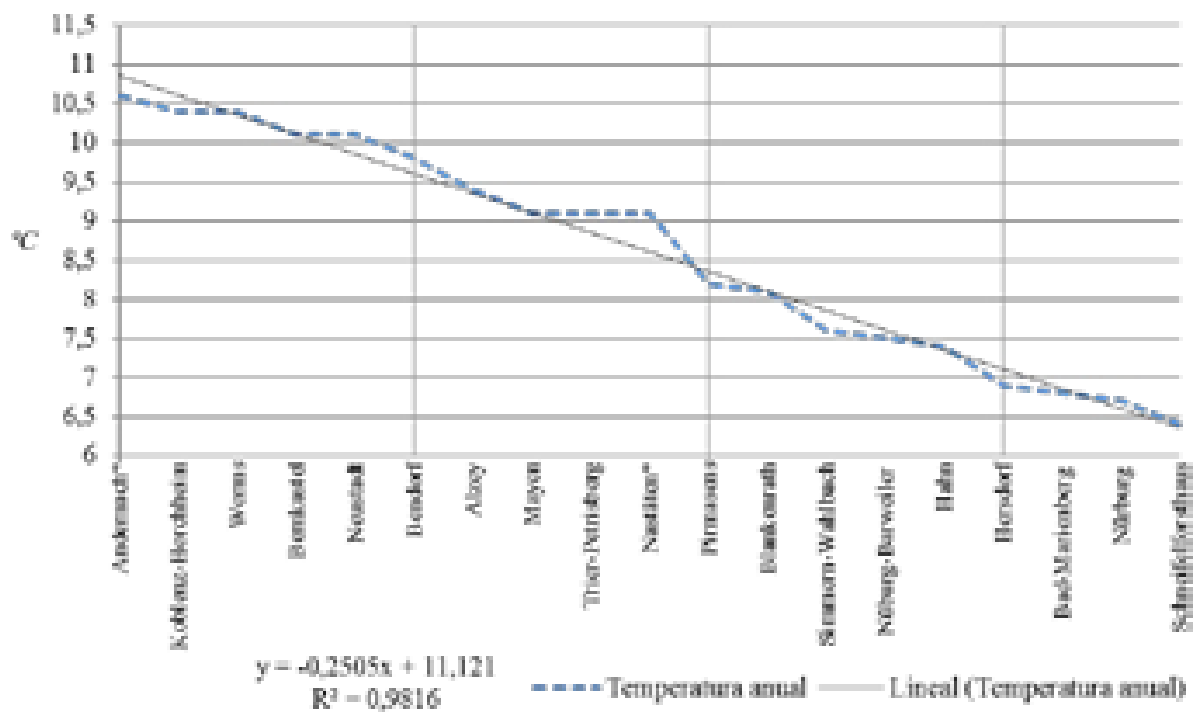

Figura 8. Cálculo del gradiente térmico vertical

Utilizándose un total de 19 estaciones de 56 posibles y partiendo desde los 75 metros de Andernach a los 649 de Schneifelforsthaus, se obtiene el gradiente aproximado para la región del Rheinland-Pfalz. De esta forma, con un $\mathrm{R}^{2}$ de 0,9816 se obtiene un gradiente vertical de $-0,2505^{\circ} \mathrm{C}$ por cada intervalo de altitud que haya entre esas estaciones, muy alejado por otra parte, de los $0,6^{\circ} \mathrm{C}$ teóricos que presuponen los manuales clásicos de climatología. A continuación, si se desea simplemente a través de la expresión " $y=a+b x$ ", se podría calcular para una altitud (y) determinada, qué temperatura (b) habría finalmente en ese punto. 


\subsection{Cálculo del gradiente térmico mensual y anual a nivel de superficie}

Como se apuntaba en la introducción, la superficie terrestre según a la altura que esté recibirá una cantidad de energía calórica concreta por parte de los rayos del sol con diferente intensidad. Además, estará condicionada por los factores que incidieran sobre ella (exposición, tipo de suelo, vegetación, usos y coberturas del suelo, vapor de agua...), variando así la temperatura final de un territorio. De esta forma, el cálculo de los gradientes, que serían estandarizadas para la región que se desee estudiar, partiría como un instrumento capaz de detectar la relación entre estaciones y los posibles desajustes por las variaciones que dichos factores externos pudieran imprimir al área de estudio. De esta forma, el siguiente paso para este trabajo aplicado sería el cálculo de cada situación atmosférica para cada intervalo de altitud de cada mes y según las posibles combinaciones entre las estaciones de los diferentes clústeres obtenidos. Finalmente, se pueden definir los comportamientos de la temperatura con una mayor precisión entre las estaciones seleccionadas que obtienen una mayor correlación estadística: descenso con la altitud, inversiones térmicas, mantenimiento de la temperatura,... Por lo tanto a continuación, se presenta la tabla 2 con los resultados obtenidos de cada extrapolación con algunas salvedades que deben ser explicadas previamente. En primer lugar, aunque para el cálculo de los gradientes lo ideal sería obtener un coeficiente de correlación superior a $0,9\left(R^{2}\right)$, no ha sido posible obtenerlo en todos los meses y para todos los intervalos, por lo que se han señalado con letra negrita aquellos resultados donde las cifran no lo alcanzan. Un segundo punto a tener en cuenta sería que para cada intervalo se ha pretendido combinar los cálculos entre estaciones de diferentes clústeres con objeto de encontrar tendencias y desajustes térmicos, es decir, descensos de la temperatura con la altitud o inversiones térmicas. Sin embargo, se ha dado el caso de que en algunos intervalos o no existe (con toda lógica) o solo se da para algunos meses concretos entre estaciones climáticas con comportamientos más diferenciados al resto. Esta situación será simbolizada con la celda oscurecida en la tabla 2. Por último, se debe prestar atención a los dos últimos cálculos realizados correspondientes a las medias anuales. El primero pertenece al de la curva de estado con todas las temperaturas medias de todas las estaciones para ese intervalo y el segundo sería el promedio anual realizando una media aritmética entre los resultados mensuales. Con esos datos solo se observan diferencias para el intervalo donde los $\mathrm{R}^{2}$ han sido más bajos de forma general (Ej. 300-400 m.s.n.m), por lo que se demuestra que hay un desajuste térmico generalizado para todo ese territorio comprendido entre esas estaciones utilizadas. 


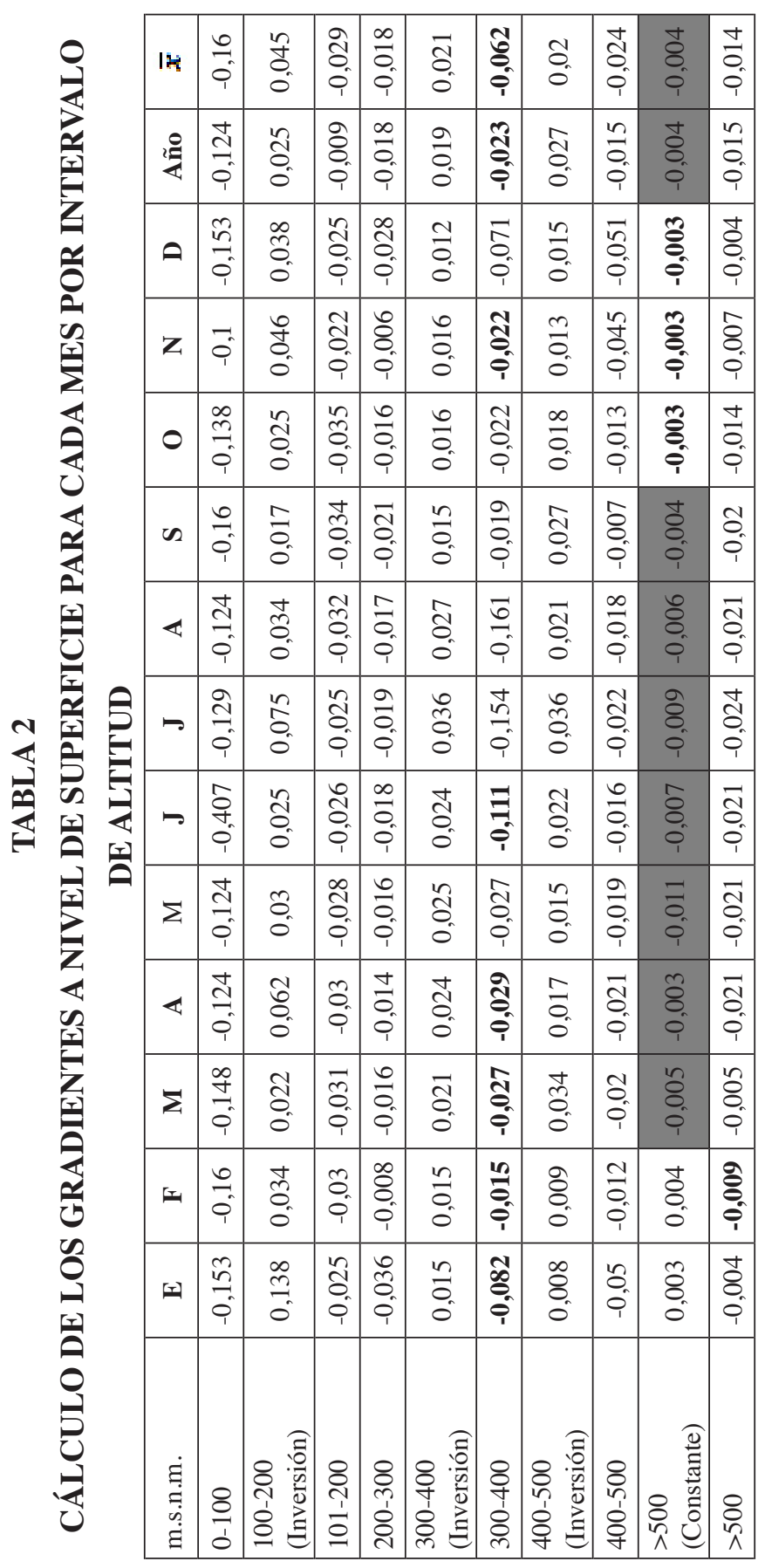


Como se puede observar en la tabla 2, existen varias posibilidades en cuanto a comportamientos del gradiente se refiere. Para el intervalo de 0-100 $\mathrm{m}$, se describe un descenso de la temperatura conforme se asciende utilizando las estaciones de Ems, Koblenz y Neuwed, siendo más acusado este en el mes de julio con $-0,407^{\circ} \mathrm{C}$ y menos en noviembre $(-0,1)$. En el siguiente intervalo (100-200), se hayan dos comportamientos contrarios según los datos que se utilicen: inversión térmica con las estaciones de Trier-Zewen y Neustadt, junto con las de Bad Dürkheim, Bad Kreuznach y Neunahr (que como ya se comentó, permanecían en los mismos clústeres); y descenso de la temperatura entre las de Bendorf, Bernkastel y Beetrich. Por otra parte, el comportamiento para las altitudes comprendidas entre 200-300 no muestra signos de desajustes termométricos y se obtienen con un coeficiente elevado entre las estaciones de Trier-Petrisberg, Montabur y Nastäten por un lado, y Alzey, Mayen, Hilgenroth y Kasel por otro. Los resultados anuales son los mismos y las variaciones no son muy elevadas, siendo en Noviembre y Febrero cercanos a una pérdida nula de calor.

La inversión térmica obtenida para el intervalo 300-400 m.s.n.m. alcanza un mayor coeficiente de correlación que el descenso con la altitud. Los mejores resultados muestran un ascenso de la temperatura entre las estaciones de Olsdorf, Rengsdorf, Hummerich (1981-2000), Niederwörresbach y con las estación del clúster más aislado en el que residía Idar-Oberstein. Aunque los valores no crecen de forma drástica, sería interesante georreferenciar y delimitar su aparición para usos agrícolas en laderas de solana alejados de los valles para favorecer los procesos fotosintéticos, por ejemplo, de la vid (Smith y Bentzen, 2011).

En la penúltima agrupación de estaciones, aparecen de nuevo dos posibles soluciones para comprender el comportamiento termométrico de la atmósfera a nivel de superficie. Para el descenso de la temperatura conforme se asciende, se obtiene un $\mathrm{R}^{2}$ elevado con las estaciones de Deuselbach, Blankenrath (1961-1980), Büchel, Hahn (1981-2000), Nürburg-Barweiler y Rupperstein. Los valores permanecen también lejos de descensos bruscos, siendo los más fuertes en los meses de Noviembre y Diciembre (cercanos a $-0,05^{\circ} \mathrm{C}$ ). Por el contrario, para las situaciones de inversión térmica se ha calculado dicho desajuste utilizando un mayor número de puntos de medición pertenecientes a cada uno de los clústeres obtenidos (algunos coincidentes con el anterior comportamiento termométrico): Büchel, Nürburg-Barweiler, Daun, Deuselbach, Rupperstein, Simmern-Wahlbach (1981-2000), Manderscheid y Hahn (1961-1981). Para el último intervalo, donde se han situado las estaciones que están emplazadas a más de 500 metros de altitud, los resultados muestran que la situación más probable estadísticamente es el descenso de la temperatura cuanto más se asciende, pero con datos muy cercanos al mantenimiento 
constante del gradiente. Los datos que mejor coeficiente obtienen son los de Weinbiet, Hersdorf, Nürburg y Schneifelforsthaus. Como ya se ha advertido, se observa como la línea del gradiente no obtiene valores muy elevados en los descensos térmicos oscilando los promedios entre $-0,014$ y $-0,015^{\circ} \mathrm{C}$

\subsection{Representación de los gradientes térmicos atmosféricos y sus tenden- cias.}

Por último, se representan en una serie de gráficos los diferentes resultados obtenidos en la tabla 2, apoyados con el cálculo de la tendencia lineal que siguen los datos y un coeficiente de correlación entre ellos, con objeto de observar qué gradientes son más correctos estadísticamente. Cada gráfica pretende mostrar el comportamiento de los puntos de cada gradiente a nivel superficial para los diferentes meses y el promedio anual en las distintas situaciones termométricas detectadas para cada intervalo para la región del Rheinland-Pfalz en general, ya no por espacios concretos. Además, será indispensable tener presente a la hora de analizar cada resultado que la correlación y la tendencia final se verán siempre afectadas por los cálculos realizados anteriormente, es decir, las líneas más fiables estadísticamente serán aquellas que posean en su representación una mayor presencia de puntos con un $\mathrm{R}^{2}$ cercano a 1 en la mayoría de los intervalos de altitud y mensualmente.

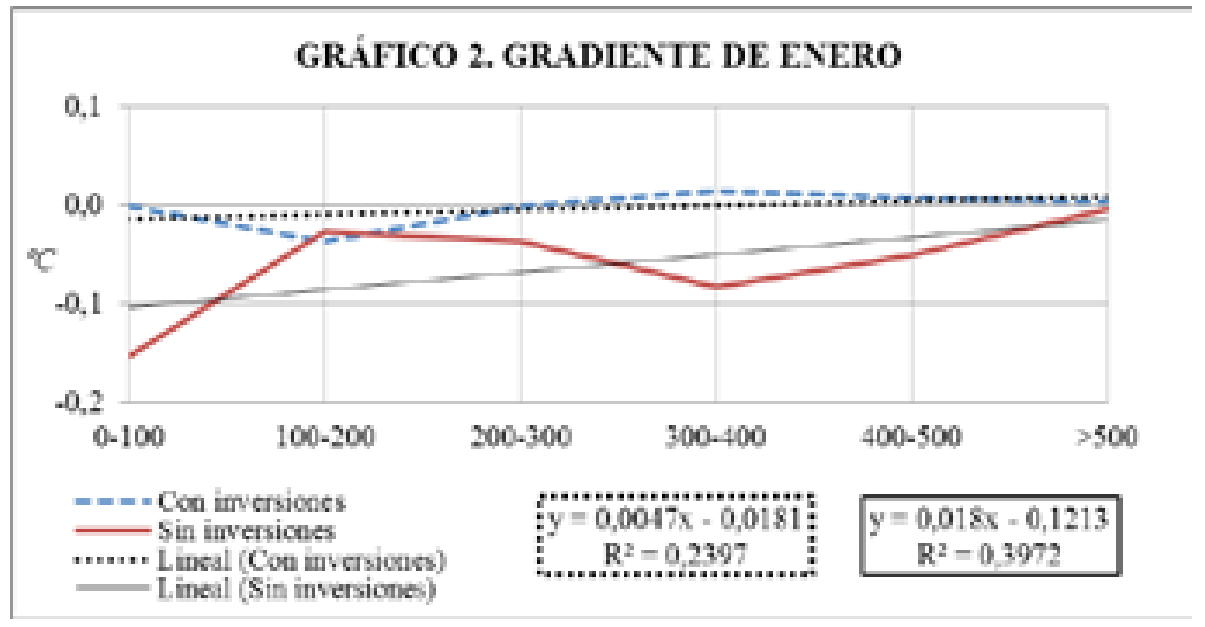



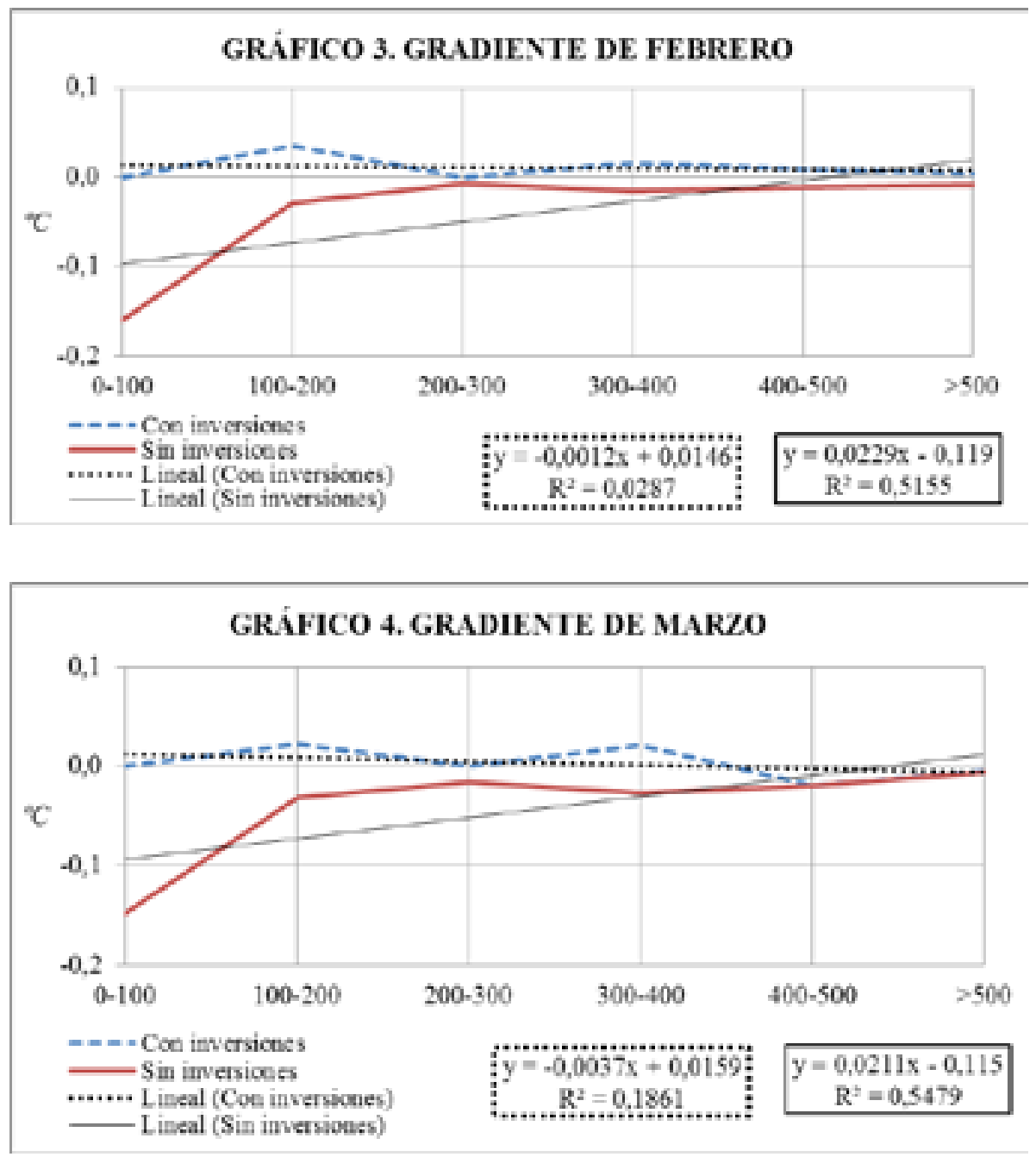

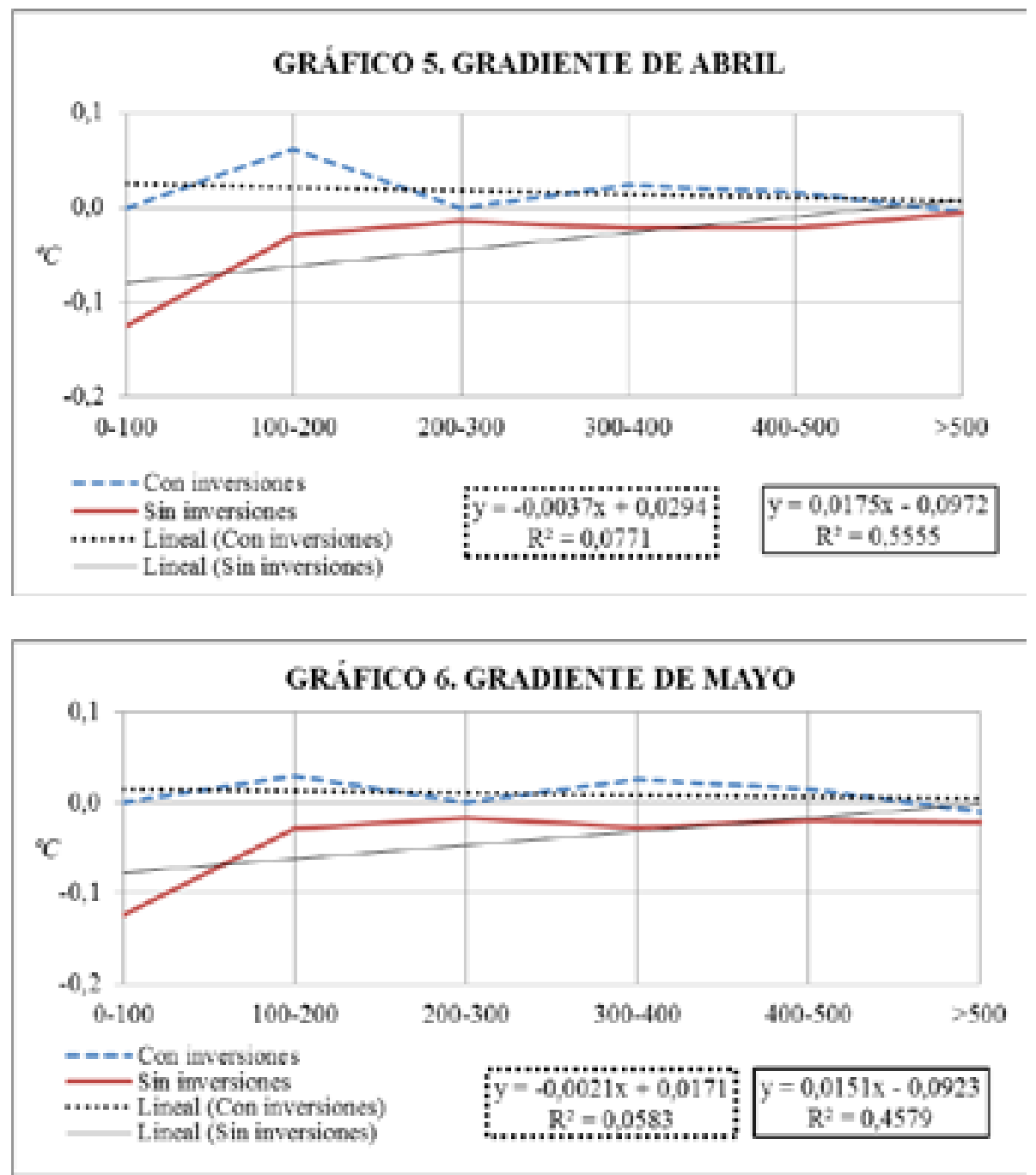

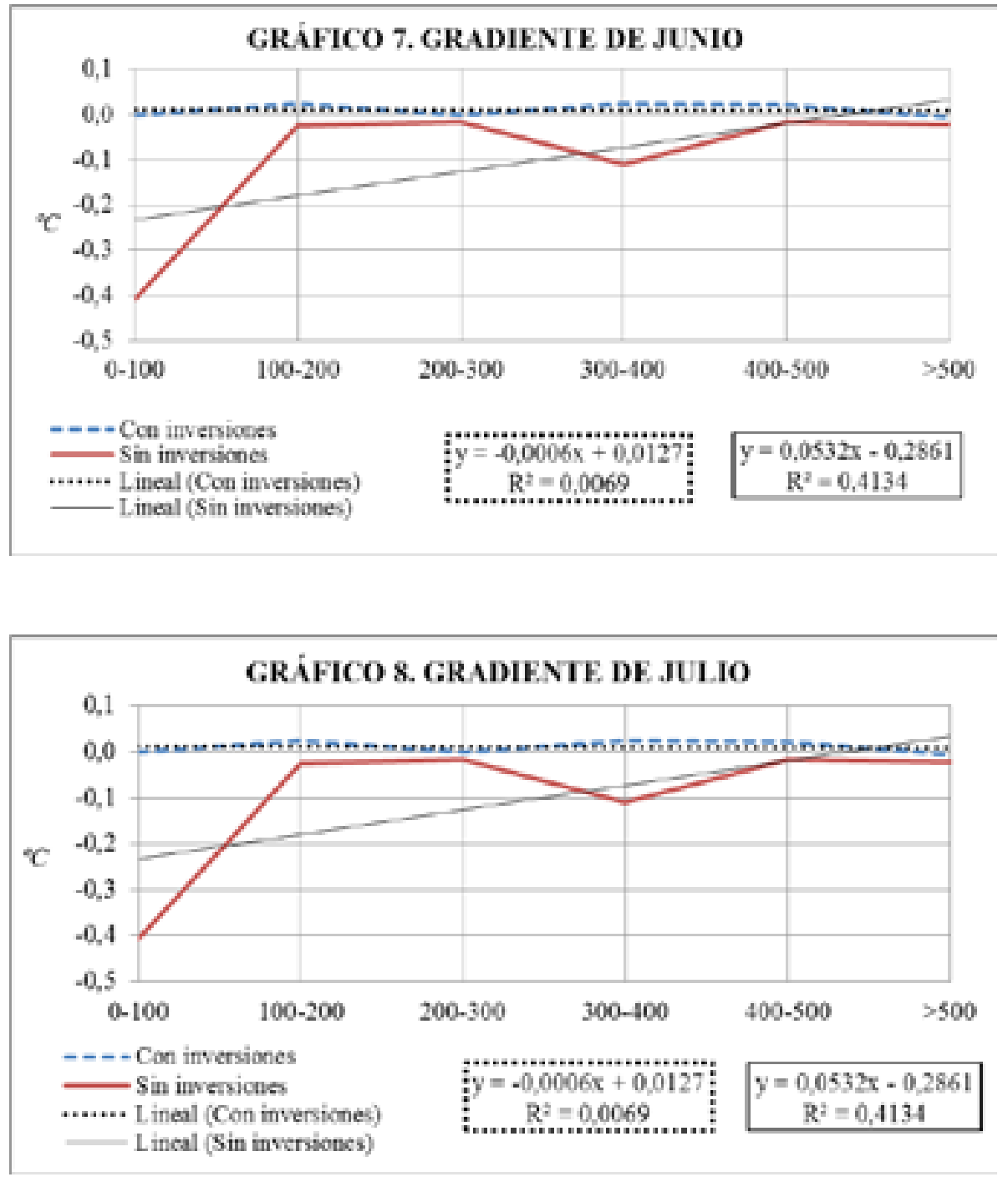

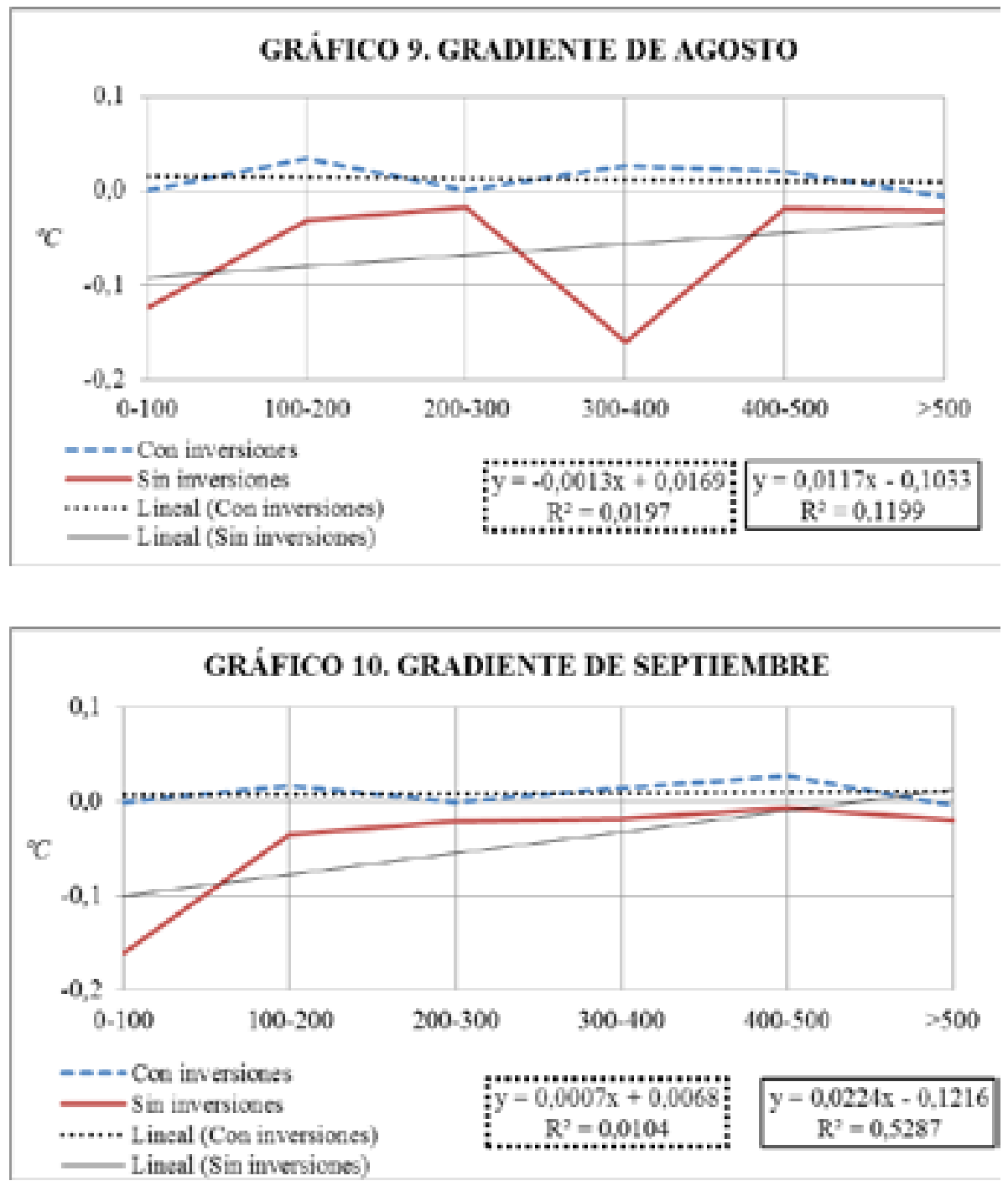

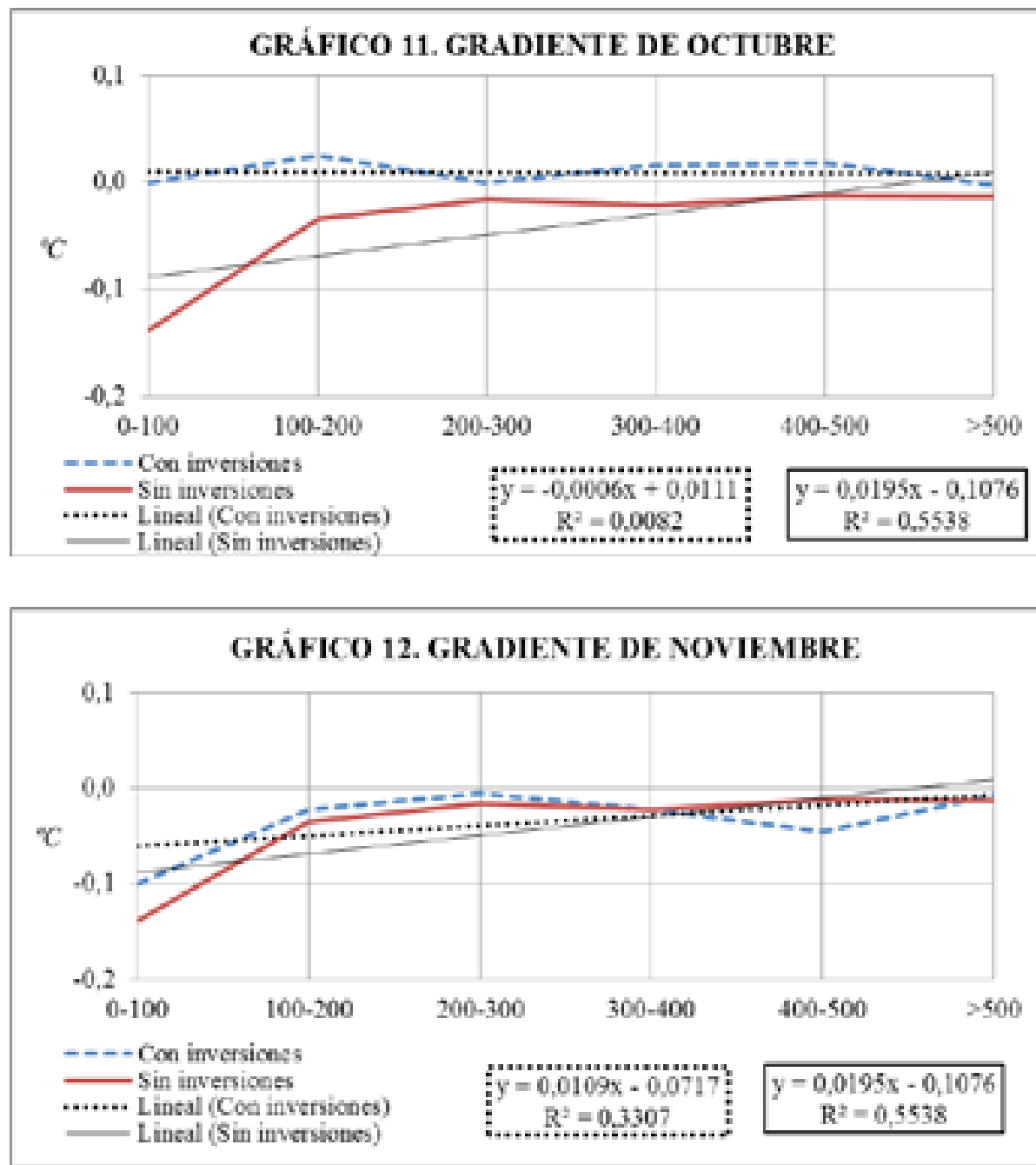


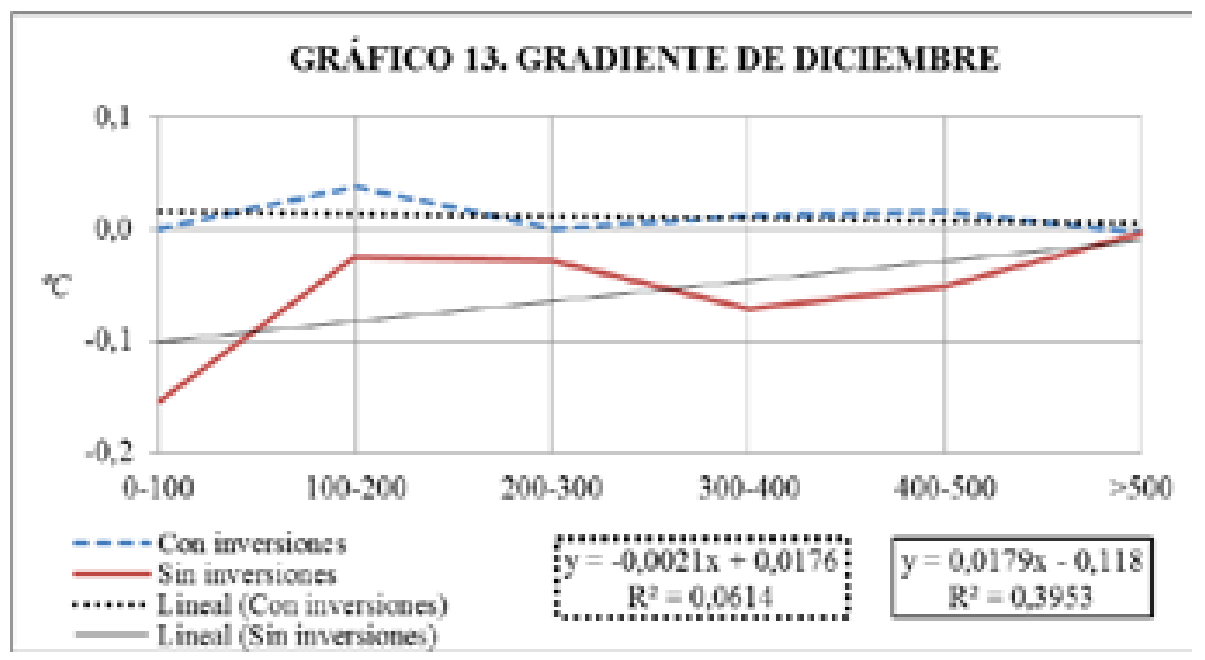

En un análisis global de las gráficas de la evolución de cada comportamiento del gradiente mensualmente, se puede advertir una idea generalizada: no existen elevadas diferencias en las pérdidas o ganancias termométricas entre intervalos por mes, pero sí son relevantes las diferencias entre el fondo del valle y la montaña. Las mayores variaciones aparecen en los meses de junio y julio en las situaciones sin inversiones térmicas con diferencias más altas entre los intervalos de 0-100 a 100-200 y desde 300-400 a más de 500 metros, donde se puede intuir la influencia de factores puntuales externos como masas de bosques, emplazamientos de las estaciones a umbría o cercanos a núcleos poblados. Con respecto a los $\mathrm{R}^{2}$, los valores más elevados son generalmente los de las situaciones donde las estaciones utilizadas la temperatura decrece con la altitud (sobre todo enero, marzo y noviembre) y, para las inversiones térmicas en las épocas del año en las que comienzan y transcurren los equinoccios (febrero-marzo-abril y septiembre-octubre-noviembre). Por el contrario, los coeficientes más bajos coinciden con los meses con temperaturas más extremas tanto cálidas como frías (diciembre-enero y junio-julio).

\section{CONCLUSIONES}

A través del cálculo de los gradientes térmicos atmosféricos a nivel de superficie, se puede alcanzar un mayor conocimiento de las situaciones térmicas concretas de un territorio, como ha sido este caso aplicado para el estado del Rheinland-Pfalz (Alemania) por intervalos de altitud, mensual y anualmente. Este instrumento es capaz de facilitar los análisis de los comportamientos concretos de la atmósfera entre los territorios comprendidos por diferentes 
estaciones climáticas con datos de temperaturas. Así, se han podido observar tendencias y desajustes por intervalos de altitud o de forma general para la región en su conjunto por meses y anualmente entre el fondo de valle o las zonas más elevadas. Por lo tanto, será una información útil para, en posteriores análisis, georreferenciar dicha información (estaciones, descenso, mantenimiento o aumento de la temperatura) con la creación de isotermas conforme a la topografía del espacio concreto a estudiar.

En el caso alemán que se ha presentado para el análisis de este estudio, se muestran pocas variaciones termométricas entre meses, pero sí diferencias entre el valle y las áreas más sobreelevadas. De esta forma, es necesario advertir dos pautas características de este territorio definidas a partir de este trabajo: i) El gradiente vertical no es ni constante (sufre numerosas inflexiones), ni generalizable a todo un territorio; ii) se aprecian situaciones de inversiones térmicas y desajustes con algunos valores en las correlaciones muy bajos provocados por factores externos ya mencionados en la literatura clásica; iii) en los meses de verano e invierno las diferencias o desajustes térmicos entre montaña y valle se hacen más notables, pudiéndose cuantificar y representar con los gradientes térmicos a nivel de superficie.

Como conclusión, se advierte de vital importancia recurrir a este tipo de análisis para detectar posibles inconvenientes térmicos que puedan provocar pérdidas económicas por problemas de localización de emplazamiento de cultivos o usos turísticos por riesgos de heladas, baja valencia ecológica de las plantas, incómodas sensaciones térmicas... En definitiva, todo ello extrapolable a otros territorios, con una aplicación directa para la Ordenación del Territorio y la investigación que gire en torno a estudios agroclimáticos, edafogeográficos o biogeográficos. 


\section{BIBLIOGRAFÍA}

CEOTMA (1996): Guía para la elaboración de estudios del medio físico: contenido y metodología. Ministerio de Obras Públicas y Urbanismo, serie: Manuales, 572 pp.

DEMANGEOT, J. (1989): Los medios “naturales” del globo. Masson, Barcelona. $256 \mathrm{pp}$.

ESCOURROU, G. (1981): Climat et environnement: Les facteurs locaux du climat. Masson, Barcelona. $182 \mathrm{pp}$.

FLOHN H. (1968): Clima y tiempo. Guadarrama, Madrid. 256 pp.

FONT TULLOT, I. (1983): Climatología de España y Portugal. Instituto Nacional de Meteorología, Madrid. 296 pp.

GEBHARDT, H., GLASER, R., RADTKE, U. y REUBER, P (2012): Physische Geographie und Humangeographie. Spektrum Akademie, 1.330 pp.

GLASER, R. (2001): Klimageschichte Mitteleuropas: 1000 Jahre Wetter, Klima, Katastrophen. Primus Verlag, Darmstadt, $227 \mathrm{pp}$.

GUNZELMANN, T. (2005): "Kulturlandschaft und Denkmalbegriff", en: Künftige Strategien der Denkmalerfassung und Denkmalforschung. Bayerisches Landesamt für Denkmalpflege, München, 3-7.

KRÄMER, C., (2009): “Der Strukturwandel im Weinbau während des 14. und 15. Jahrhundertsam Beispiel der Weinanbaugebiete im Vorland der Schwäbischen Alb". Landnutzung und Landschaftsentwicklung im deutschen Südwesten, 2140.

PÉDELABORDE, P. (1982): Introduction à l'étude scientifique du climat. SEDES, Paris. 353 pp.

PETIT, C. KONOLD, W. y HÖCHTL, F. (2012): "Historic terraced vineyards: impressive witnesses of vernacular architecture". Landscape History, 33 (1), 5-28.

SENCIALES, J.M. (2007): El clima en la provincia de Málaga, en: Atlas Hidrogeológico de la provincia de Málaga. Tomo 2, 143-148. (J.J. DURÁN). Madrid: Instituto Geológico y Minero de España; Diputación de Málaga.

STRAHLER A. H. \& STRAHLER A. N. (1989): Geografía Física. Omega. 562 pp.

UNWIN, T. (1996): Wine and the Vine: an historical geography of viticulture and the wine trade. New York. 414 pp.

\section{WEBGRAFÍA}

www.dwd.de (10/2013)

www.lgb-rlp.de (10/2013) 